\title{
A generalization of the van-der-Pol oscillator underlies active signal amplification in Drosophila hearing
}

\author{
Journal Article \\ Author(s): \\ Stoop, R.; Kern, A.; Goepfert, M.C.; Smirnov, D.A.; Dikanev, T.V.; Bezrucko, B.P. \\ Publication date: \\ 2006-08 \\ Permanent link: \\ https://doi.org/10.3929/ethz-b-000036569 \\ Rights / license: \\ In Copyright - Non-Commercial Use Permitted \\ Originally published in: \\ European Biophysics Journal 35(6), https://doi.org/10.1007/s00249-006-0059-5
}




\section{A generalization of the van-der-Pol oscillator underlies active signal amplification in Drosophila hearing}

Received: 6 February 2006/ Revised: 21 February 2006/ Accepted: 15 March 2006 / Published online: 13 April 2006 (C) EBSA 2006

\begin{abstract}
The antennal hearing organs of the fruit fly Drosophila melanogaster boost their sensitivity by an active mechanical process that, analogous to the cochlear amplifier of vertebrates, resides in the motility of mechanosensory cells. This process nonlinearly improves the sensitivity of hearing and occasionally gives rise to self-sustained oscillations in the absence of sound. Time series analysis of self-sustained oscillations now unveils that the underlying dynamical system is well described by a generalization of the van-der-Pol oscillator. From the dynamic equations, the underlying amplification dynamics can explicitly be derived. According to the model, oscillations emerge from a combination of negative damping, which reflects active amplification, and a nonlinear restoring force that dictates the amplitude of the oscillations. Hence, active amplification in fly hearing seems to rely on the negative damping mechanism initially proposed for the cochlear amplifier of vertebrates.
\end{abstract}

The cochlear amplifier is a fundamental, generally accepted concept in cochlear mechanics, having a large impact on our understanding of how hearing works. This concept, first brought forward by Gold (1948),

R. Stoop $(\bowtie) \cdot$ A. Kern

Institute of Neuroinformatics, University/ETH Zürich,

Winterthurerstr. 190, 8057 Zurich, Switzerland

E-mail: ruedi@ini.phys.ethz.ch

M. C. Göpfert

Volkswagen Foundation Research Group, Institute of Zoology, University of Cologne, 50923 Cologne, Germany

D. A. Smirnov

Institute of Radioengineering and Electronics of R. Acad. Sci., Saratov Branch, Saratov 410019, Russia

T. V. Dikanev $\cdot$ B. P. Bezrucko

Department of Nonlinear Processes, Saratov State University, Saratov 410026, Russia posits that an active mechanical process improves the mechanical performance of the ear (Robles and Ruggero 2001). Active components consume power other than the signal itself, to provide amplification. Until recently, the study of this amplificatory process has been restricted to the ears of vertebrates, where the high complexity and the limited accessibility of the auditory system complicate the in situ investigation of the mechanisms involved. This limitation has hampered the validation of cochlear models that have been devised (e.g., de Boer 1996; Kern and Stoop 2003). The hearing organs of certain insects have recently been shown to exhibit signal processing characteristics similar to the mammalian cochlea by using active amplification: the ears of these insects are able to actively amplify incoming stimuli, display a pronounced compressive nonlinearity, exhibit power gain, and are able to generate self-sustained oscillations in the absence of sound (Göpfert and Robert 2001, 2003; Göpfert et al. 2005). In both vertebrates and insects, the mechanism that promotes this amplification resides in the motility of auditory mechanosensory cells, i.e. vertebrate hair cells and insect chordotonal neurons. Both types of cells are developmentally derived by homologous genes and share similar transduction machineries, pointing to a common evolutionary origin (Boekhoff-Falk 2005). In line with such an evolutionary scenario, it seems possible that also the fundamental mechanism of active amplification in the ears of insects and vertebrates is evolutionary conserved (Robert and Göpfert 2002).

Since insect hearing organs are located on the body surface (head, thorax, legs etc.), they are accessible to non-invasive examination. Moreover, because the external sound receiver is often directly coupled to the auditory sense cells, insect auditory systems can be expected to provide profound experimental and theoretical insights into the in situ mechanics of motile sense cells and their impact on the mechanical performance of the ear. Such information is technically relevant: providing natural examples of refined active sensors, the minuscule ears of insects promise inspiration for the design of 
nanoscale artificial analogues. In this contribution, we model self-sustained oscillations of the antennal ear of the fruit fly Drosophila melanogaster. By using timeseries analysis methods, we reconstruct the generating differential equation and we show that the amplificatory process is well described by a generalization of the van-der-Pol equation. The fly's auditory system is shown to be driven by a regenerative amplifier, as was initially proposed by Gold (1948) for the cochlear amplifier of vertebrates.

The basic acoustic stimulus to which Drosophila responds is the courtship song, produced by the courting male, which is a sinusoidal sound wave having an average frequency of $160 \mathrm{~Hz}$, with considerable variation between males. From the antenna's distal part that forms the external sound receiver, hearing is mediated by directly connected mechanosensory neurons (Göpfert and Robert 2003). These neurons actively modulate the receiver's mechanics and, occasionally, give rise to selfsustained receiver oscillations (SO). In Drosophila, SO occasionally occur spontaneously, i.e. when the physiological condition of the animal deteriorates, and upon thoracic injection of dimethylsulphoxide (DMSO) (Göpfert and Robert 2001). For both the cases, the physiological mechanism that drives the fly's auditory system into the oscillatory regime remains unclear. Yet, because spontaneously occurring and DMSO-induced oscillations are both physiologically vulnerable and display similar waveforms, they can be used to probe active amplification in the fly's antennal ear (Göpfert and Robert 2001). As DMSO reliably induces oscillations in the ear of the wild-type Drosophila (Oregon R strain), we have focused our analysis on the DMSO-induced SO, where our measuring device consisted of a computercontrolled Ploytec PSV-400 scanning laser Doppler vibrometer with a OFV-5000 scanning head, and a controller. All measurements were taken on the distal part of the receiver, i.e. the tip of the antenna's arista [for a detailed technical description, see (Göpfert et al. 2005)].

Self-sustained receiver oscillations (SO) emerge some 10 min after the administration of DMSO, beginning at a frequency around $130 \mathrm{~Hz}$. During the following processes, this frequency continually decreases. Already a few minutes later (at about $100 \mathrm{~Hz}$ ), the $\mathrm{SO}$ attain their fully developed relaxation-oscillation like waveform (Fig. 1b; Göpfert and Robert 2003). For a longer period, this waveform is maintained. From there, the SO abruptly change into a temporally extended state with reduced amplitudes (Fig. 1c), from where, again rather abruptly, the final sinusoidal shape is attained (Fig. 1d). DMSO evoked SO can last up to 1-1.5 h until they disappear with the animal's death (Göpfert and Robert 2003). Their typical shapes (see Fig. 1) are reminiscent of limit-cycle oscillations generated by a van-der-Pol type oscillator,

$\ddot{x}-\mu\left(1-x^{2}\right) \dot{x}+x=0$,

where $x$ is identified with the receiver's vibrational position and where the control parameter $\mu>0$ is slowly decreased in order to account for the changes of SO shapes during time. It is well known that at $\mu=0$, the van-der-Pol oscillator undergoes a Hopf bifurcation: for $\mu>0$, stable limit cycles emerge that can be interpreted as negative damping (i.e. amplification). A detailed examination, by comparing the onsets and extents of the upward and downward excursions within one period of the experimental data, reveals a pronounced asymmetry (see Fig. 1b), which requires a more general model for SO generation than the standard van-der-Pol system.

In order to capture this asymmetry, we used a generalization

$\ddot{x}+P_{n}(x) \dot{x}+P_{m}(x)=0$,

of the van-der-Pol oscillator, where $P_{n}(x)$ and $P_{m}(x)$ describe polynomials of yet unknown orders $n$ and $m$, respectively. From the point of Physics, $P_{n}(x)$ describes a nonlinear, and possibly negative, damping, whereas $P_{m}(x)$ accounts for a nonlinear restoring force. Our objective is to determine the orders $n, m$ and the polynomial coefficients that yield the optimal reproduction of the experimental data. We expect that for a proper model, the polynomial orders $n$ and $m$ are unambiguously determined, and only the variation of the coefficients will account for the observed changes in the SO shapes over time.

From the measurements, we are provided with the receiver's vibration velocities time series. Our reported results are based upon a typical, reproducible, time series of 160,000 data points, obtained from one individual fly. Stationarity requires to dissect the time series into subfiles of 4,000 data points. In Fig. 1, the characteristic subfiles from the temporal evolution are shown. In order to determine the optimal model, the receiver's displacements and accelerations are required. This is obtained by numerical integration and differentiation of the data, respectively. For both the cases, characteristic difficulties must be overcome. In the case of the receiver position, slow changes in the mean velocity induce a noticeable drift in the computed locations. This drift can be eliminated by approximating the computed locations by a polynomial in the least-squares sense and subtracting the polynomial values from the location values. Using a polynomial of 20th order, the nonlinear trends are annihilated, including the linear and the quadratic contributions. Unfortunately, high-order polynomials induce strong oscillations in the vicinity of the beginning and end of the time series. For the further analysis, these parts of the time series must therefore be excluded. In the case of the numerical differentiation, the measured time series must be smoothed in order to reduce the effects of noise. By applying a first-order SavitskyGolay filter (Savitsky and Golay 1964), this can be achieved in an efficient way.

As the basis for our fit to the data, we consider polynomials of the form

$f(x, \dot{x})=-P_{n}(x) \dot{x}-P_{m}(x)$. 
Fig. 1 Self-sustained oscillations $(\mathrm{mm} / \mathrm{s})$ of the Drosophila hearing sensor (velocity measurements), a $10 \mathrm{~min}$, b $20 \mathrm{~min}$, c $30 \mathrm{~min}$, d 34 min after dimethylsulphoxide (DMSO) injection
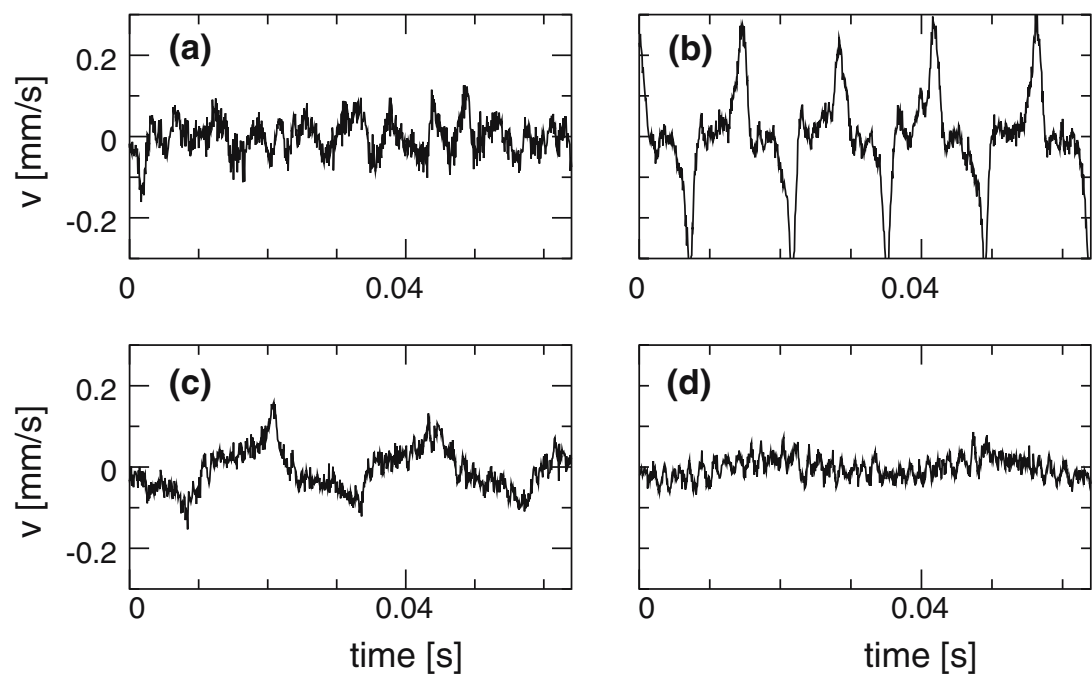

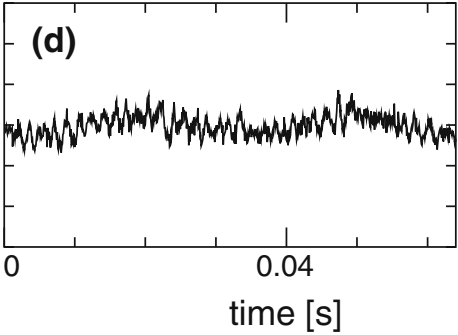

In this notation, the differential equation takes the form $\ddot{x}=f(x, \dot{x})$. In order to determine the optimal polynomial orders, for each $(n, m)$ order model the polynomial coefficients that minimize the squared error

$\epsilon_{n, m}^{2}=\sum_{i=1}^{N}\left(\ddot{x}\left(t_{i}\right)-f\left(x\left(t_{i}\right), \dot{x}\left(t_{i}\right)\right)\right)^{2}$,

are determined, whereby the time series $\left\{\ddot{x}\left(t_{i}\right)\right\}$ are normalized to have unit variance. Since the time series are non-stationary, the time steps $t_{i}$ at which $\dot{x}\left(t_{i}\right)$ is measured should be restricted to a quasistationary subset of the entire time series. The lengths $N$ of these subsets ( $\sim 4,000$ data points) were found to be sufficiently large for polynomial fitting being reliably performed.

It is observed that the error $\varepsilon_{n, m}^{2}$ saturates for $n=2$ and $m=5$ (Fig. 2). A further increase of $\{n, m\}$ does not reduce $\varepsilon_{n, m}^{2}$. The emergence of such a conspicuous saturation point is exceptional: it indicates that the

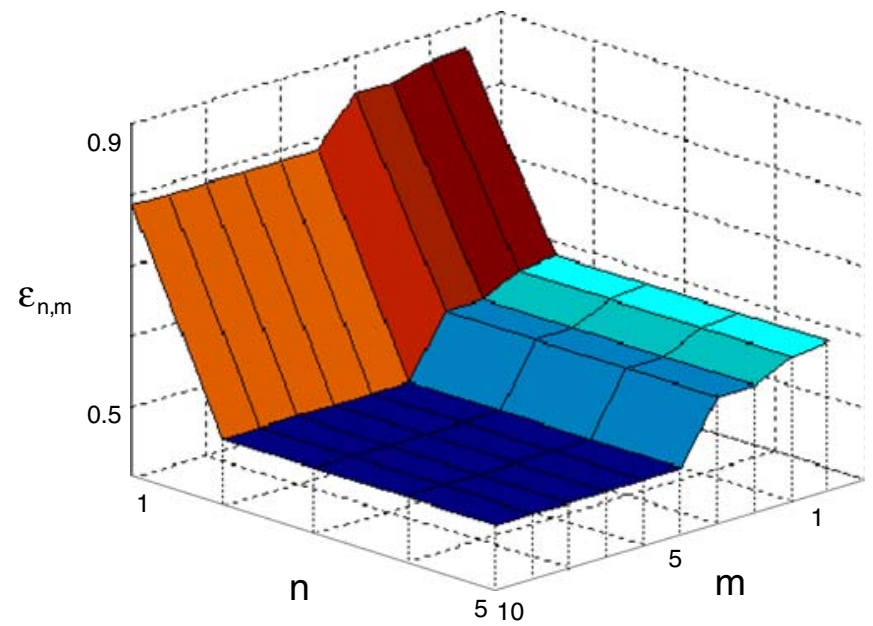

Fig. 2 Root-mean-squared error $\varepsilon_{n, m}$ of the model fitting Eq. 4, showing a precipitous decay and saturation of the error around the orders $n=2$ and $m=5$ of the polynomial approximation model structure (2) faithfully reproduces the data. It could be argued that the relatively high noise level prevents the error $\varepsilon_{n, m}^{2}$ from decreasing any further. In the absence of noise, the errors might thus gradually decrease with increasing $\{n, m\}$. Fortunately, the rapid decay of $\varepsilon_{n, m}^{2}$ before saturation provides strong evidence against this view, indicating that our modeling is realistic indeed. Moreover, noise-cleaned (Kern et al. 2000) experimental data reveal a basically unchanged decay behavior, corroborating the validity of the obtained optimal polynomial orders.

A comparison between the realizations of time series by the model and the measurements supports the validity of our approach. For the fully developed SO (after 20 min, see Fig. 3), the comparison reveals that the measured velocities are faithfully reproduced. This is further illustrated in Fig. 4, where the modeled and the measured data are compared in the phase plane $(x, \dot{x})$, whereby the positions $x$ were obtained by numerical integration from the measured velocities. Similar observations emerge for time series recorded at 10, 30, and $34 \mathrm{~min}$, respectively, after DMSO injection.

The shapes of the polynomials $P_{n}(x)$ and $P_{m}(x)$ reflect the asymmetry of the observed receiver oscillations, specifically when SO are fully developed (cf. Fig. 1b). The asymmetry of $P_{n}(x)$ and, in particular, $P_{m}(x)$ (see Fig. 5b) becomes effective at large displacements and may have its origin in structural-mechanical properties of the antenna. An enlightening interpretation of the amplification dynamics can be given for the behavior around zero displacement position $x \cong 0$, where $P_{n}(x)$ attains negative values for small displacements $x$ (Fig. 5a). Since $P_{n}(x)$ represents a nonlinear damping, $P_{n}(x)<0$ implies that energy is injected into the system. This is a characteristic feature of an active amplification process. Around $x=0$, the nonlinear restoring force $P_{m}(x)$, together with its first and second derivatives, are relatively small. This implies that for small receiver displacements, virtually no restoring force is present. By means of the negative damping term, the system is thus 


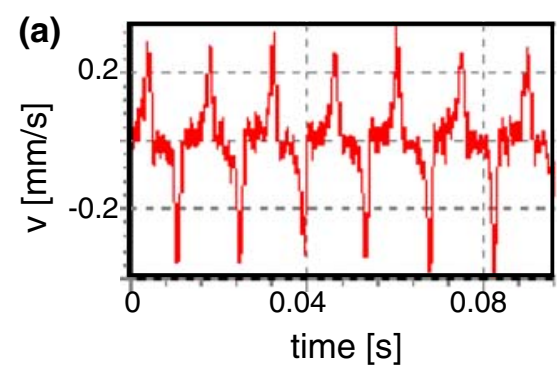

Fig. 3 a Receiver's vibration velocity $(\mathrm{mm} / \mathrm{s}), 20 \mathrm{~min}$ after DMSO injection [fully developed self-sustained receiver oscillations (SO)]. b Approximating time series generated by the model (2), using $n=2$

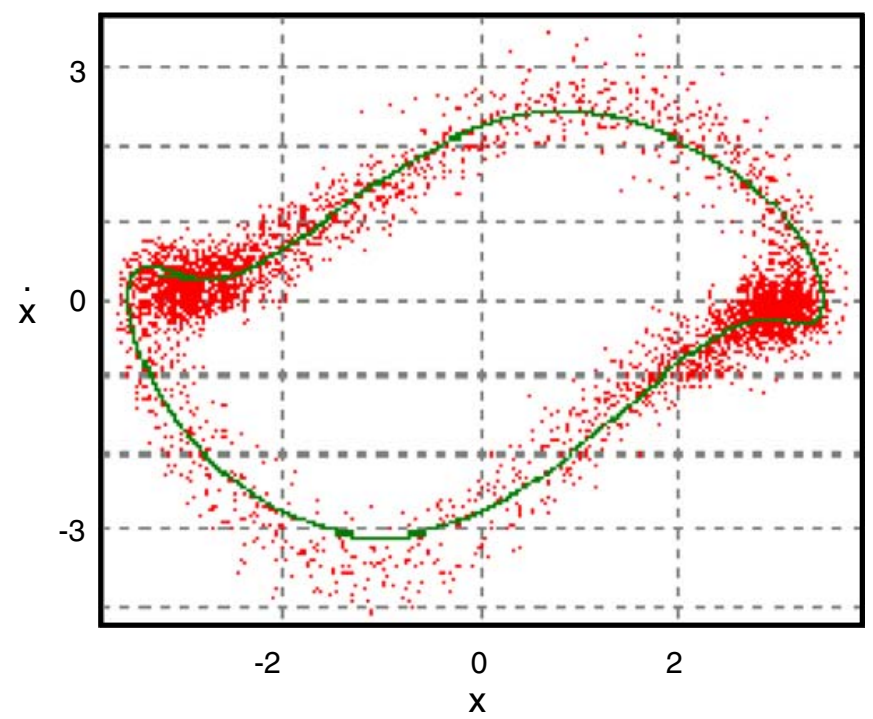

Fig. 4 Phase-space representation of measured (dots) and modeled (solid line) receiver vibrations (at fully developed SO). Units: $x$ : $\left(10^{2} \mathrm{~mm}\right), y:\left(10^{-1} \mathrm{~mm} / \mathrm{s}\right)$

easily driven out to relatively large amplitudes. Thirty minutes after administration of DMSO (Fig. 1c, d), the nonlinear contributions to damping and restoring force have decayed. In particular, the range where the damping is negative has decreased (Fig. 1c) and finally vanished (Fig. 1d), in agreement with the observed reduction in SO amplitude (see Fig. 1). In the absence of pronounced SO (Fig. 1a, c, d), the restoring force

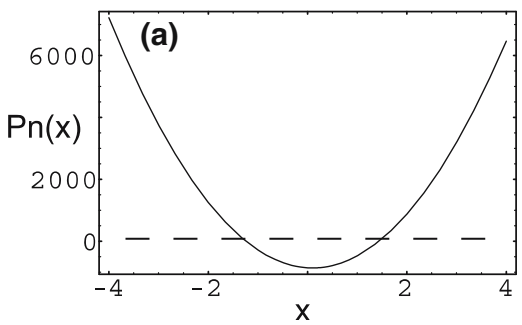

Fig. 5 Fully developed SO 20 min after DMSO injection, approximated by polynomials of degrees $n=2$ and $m=5$. a Nonlinear damping term $P_{n}(x)$, showing negative damping around the origin $\left(P_{n}(x)<0\right)$. b Nonlinear restoring force $P_{m}(x)$, displaying a (b)

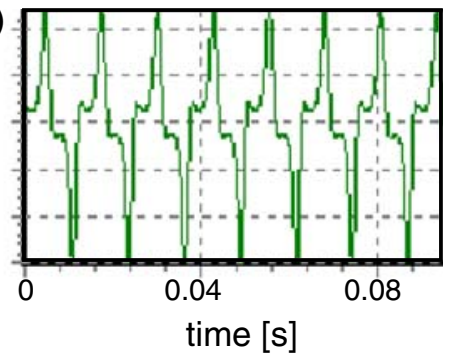

and $m=5$. Approximating polynomials are $P_{n}(x)=-8.602 \times 10^{2}-$ $9.481 \times 10^{5} x+4.811 \times 10^{10} x^{2}, P_{m}(x)=31.98+2.023 \times 10^{6} x-$ $7.85 \times 10^{8} x^{2}-7.474 \times 10^{13} x^{3}+1.501 \times 10^{15} x^{4}+7.488 \times 10^{20} x^{5}$

function $P_{m}(x)$ obtains an approximately linear characteristic with a very small slope. At the same time, the damping term remains small. As a consequence, weak stimuli will still be sufficient to elicit considerable antennal vibrations. Hence, even when the amplifier is in a stable state, where limit cycles do not occur, the sensor can be very sensitive. Small parameter changes are sufficient to render the damping term negative and to lead to an amplification of incoming vibrations. The evaluation of the temporal evolution of the polynomials $P_{m}(x), P_{n}(x)$ from subfile to subfile yields that the polynomial orders are preserved, whereas the coefficients change in time. This is remarkable in particular in the case of $P_{m}(x)$, as it indicates that this term comprises more than just mechanical antenna properties. A more than formal interpretation of the process in terms of mechanical quantities would therefore require particular care. During the evolution, the general tendency of the coefficients moduli is to decrease, in an approximately linear fashion. In spite of this fact, the fully developed oscillatory state is maintained for a long time. In the present example, the oscillatory amplitudes and the shapes of the polynomials quite abruptly flatten out after $28 \mathrm{~min}$, where the stiffness term seems to slightly precede the damping term. The decrease appears to conserve the fine balance between the stiffness and damping term that has been discussed for the fully developed case.

Our results may be compared to measurements of active hair-bundle oscillations of vertebrate hair cells, pointing to a slight difference in the mechanisms

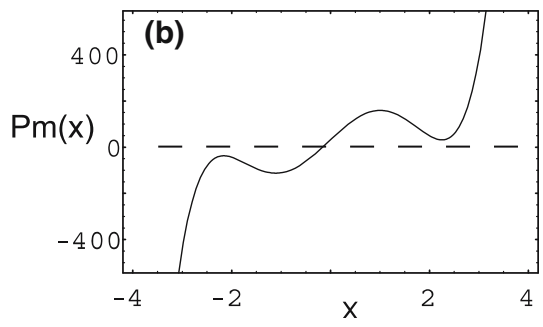

noticeable asymmetry. Its derivative $P_{m}^{\prime}(x)$ indicates areas of negative stiffness $\left(P_{m}^{\prime}(x)<0\right)$. Units: $x:\left(10^{2}\{\mathrm{~mm}), y:\left(\mathrm{s}^{-1}\right)\right.$, $\left(\left\{\mathrm{mm} \mathrm{s}^{-2}\right)\right.$, respectively 
involved. Active hair bundle oscillations promote active amplification in nonmammalian and, possibly, mammalian vertebrates (Chan and Hudspeth 2005; Kennedy et al. 2005; Martin et al. 2000). Mechanical stimulation experiments performed on isolated hair bundles have shown that the bundle stiffness may become negative for small bundle displacements (Kennedy et al. 2005; Martin et al. 2000). In this case, bundle stiffness has its origin in amplification-promoting ion-channel dynamics. For the Drosophila ear, our model suggests that amplification emerges from negative damping; only at intermediate displacements, regions of negative stiffness (i.e., where the restoring force decreases with displacement) are found (see Fig. 5b).

Recently, it was proposed that active amplification in the mammalian hearing system is governed by the (nonlinear) Stuart-Landau equation (Kuramoto 2003) valid around a Hopf bifurcation point (Choe et al. 1998; Camalet et al. 2000; Eguíluz et al. 2000). By fixing the Hopf parameter to (Duke and Jülicher 2003; Magnasco 2003) or below (Kern and Stoop 2003; Stoop and Kern 2004) the bifurcation point, salient characteristics of cochlear mechanics such as responses to single and multi-frequency tones could be reproduced (Kern and Stoop 2003; Camalet et al. 2000; Eguíluz et al. 2000; Duke and Jülicher 2003; Magnasco 2003; Stoop and Kern 2004; Jülicher et al. 2001). Because the van-der-Pol system (Eq. 1), as well as our generalized higher-order nonlinear oscillator, exhibit a Hopf bifurcation at $\mu=0$, they show compatible amplification properties. Active amplification using Hopf nonlinearities thus appears to be a general mechanism in hearing.

Finally, a comparison with approaches of modeling spontaneous otoacoustic emissions (SOAE) of vertebrate ears by means of active nonlinear oscillators can be made. It was recently shown that in order to generate the correct exponential relaxation part behavior of SOAE, the simplest van-der-Pol oscillator variant (1) is insufficient (Sisto and Moleti 1999). For a correction, the damping coefficient term $\left(1-x^{2}\right)$ was changed into the form $\left(\frac{c}{\left(\left\langle x^{2}\right\rangle\right)^{m}}-b\right)$, where $c, b, m$ are positive numbers and $\langle\cdot\rangle$ denotes the average over many cycles of the inherent oscillations of the system. The fractional term has the effect that the nonlinear active amplification term (negative damping) grows significantly in the neighborhood of $x=0$, whereas linear damping dominates for larger amplitudes. This yields the experimentally observed exponential relaxation behavior at intermediate relaxation time. For the implementation of the also observed initial saturation regime, an additional (negative quadratic) term is required. Figure 5 and a numerical check show that these experimental features are already contained in our model.

Active amplification in the ear of $D$. melanogaster may generate self-sustained oscillations in the absence of sound. The shape and temporal pattern of these oscillations are faithfully described by a generalization of the van-der-Pol equation, where the validity of the model derived is supported by the saturation of the model fit to the experimental data. From our modeling, it emerges that the observed active amplification results from the combination of negative damping, which is quadratic in the displacement, and a strongly nonlinear restoring force that has two positions of minimal absolute size at nonzero displacement. Within the area of negative damping, the restoring force is almost linear. In this region, negative damping will amplify the receiver's vibrations, even if the resulting displacement is too small to cross the local absolute size extrema of the restoring force. If the latter can be crossed, an oscillation displaying a characteristic displacement (at a frequency that depends on the mechanical properties of the antenna) is maintained. This seems to be an efficient way of combining high sensitivity with robustness to noise. Antenna geometry finally may be responsible for the observed asymmetry of the restoring force (visible upon close inspection of Fig. 5b).

The model described here captures several characteristics of vertebrate SOAEs, indicating that our approach may be useful for analyzing the Physics of the cochlear amplifier as well. In Drosophila, insights into the Physics of auditory amplification are important: because the antennal ear of the fly is a preferred model system to genetically dissect the processes that bring about hearing, our work provides a theoretical framework that may help to explore the integrated function of an ear.

\section{References}

Boekhoff-Falk G (2005) Hearing in Drosophila: development of Johnston's organ and emerging parallels to vertebrate ear development. Dev Dyn 232:550-558

de Boer E (1996) Mechanics of the cochlea: modeling efforts. In: Dallos P, Popper A, Fay R (eds) The Cochlea. Springer Handbook of Auditory Research, Springer, Berlin Heidelberg New York

Camalet S, Duke T, Jülicher F, Prost J (2000) Auditory sensitivity provided by self-tuned critical oscillations of hair cells. Proc Natl Acad Sci USA 97:3183-3188

Chan DK, Hudspeth AJ (2005) $\mathrm{Ca}^{2+}$ current-driven nonlinear amplification by the mammalian cochlea in vitro. Nat Neurosci 8:149-155

Choe Y, Magnasco MO, Hudspeth AJ (1998) A model for amplification of hair-bundle motion by cyclical binding of $\mathrm{Ca}^{2+}$ to mechanoelectrical-transduction channels. Proc Natl Acad Sci USA 95:15321-15326

Duke T, Jülicher F (2003) Active traveling wave in the cochlea. Phys Rev Lett 90:158101-1-158101-4

Eguíluz VM, Ospeck M, Choe Y, Hudspeth AJ, Magnasco MO (2000) Essential nonlinearities in hearing. Phys Rev Lett 84:5232-5235

Gold T (1948) Hearing ii: the physical basis of the action of the cochlea. Proc R Soc Lond B 135:492-498

Göpfert MC, Robert D (2001) Active auditory mechanics in mosquitoes. Proc R Soc Lond B 268:333-339

Göpfert MC, Robert D (2003) Motion generation by Drosophila mechanosensory neurons. Proc Natl Acad Sci USA 100:5514 5519

Göpfert MC, Humpfries ADL, Albert JT, Robert D, Hendrich O (2005) Power gain exhibited by motile neurons in Drosophila ears. Proc Natl Acad Sci USA 102:325-330 
Jülicher F, Andor D, Duke T (2001) Physical basis of two-tone interference in hearing. Proc Natl Acad Sci USA 98:9080-9085

Kennedy HJ, Crawford AC, Fettiplace R (2005) Force generation by mammalian hair bundles supports a role in cochlear amplification. Nature 433:880-883

Kern A, Stoop R (2003) Essential role of couplings between hearing nonlinearities. Phys Rev Lett 91:128101-1-128101-4

Kern A, Steeb W-H, Stoop R (2000) Projective noise cleaning with dynamic neighborhood selection. Int J Mod Phys C 11:125-146

Kuramoto Y (2003) Chemical oscillations, waves, and turbulence. Dover Publications, Dover

Magnasco MO (2003) A wave traveling over a Hopf instability shapes the cochlear tuning curve. Phys Rev Lett 90:058101-1058101-4
Martin P, Mehta AD, Hudspeth AJ (2000) Negative hair-bundle stiffness betrays a mechanism for mechanical amplification by the hair cell. Proc Natl Acad Sci USA 97:12026-12031

Robert D, Göpfert MC (2002) Novel schemes for hearing and orientation in insects. Curr Opin Neurobiol 12:715-720

Robles L, Ruggero M (2001) Mechanics of the mammalian cochlea. Physiol Rev 81:1305-1352

Savitsky A, Golay MJE (1964) Smoothing and differentiation of data by simplified least square procedures. Anal Chem 36:1627-1639

Sisto R, Moleti A (1999) Modeling otoacoustic emissions by active nonlinear oscillators. J Acoust Soc Am 106:1893-1906

Stoop R, Kern A (2004) Two-tone suppression and combination tone generation as computations performed by the Hopf cochlea. Phys Rev Lett 93:268103-1-268103-4 